\title{
DOSSIER
}

\section{Introducción al dossier. Aportes al debate de enfoques y métodos de investigación social.}

Marĩa Andrea Benítez1

\begin{abstract}
${ }^{1}$ Arquitecta UNNE. Magister en Epistemologìa y Metodología de la investigación científica UNNE. Candidata Doctoral en Antropología social UNaM. Investigadora de la UNNE. Prof Adjunto a cargo de Metodología de la Investigación en ciencias sociales en Lic. RRLL de la FCE UNNE; y JTP de Teoría del Diseño y la Gestión Urbana/Sociología Urbana en FAU UNNE.
\end{abstract}

Pese a ser un campo consolidado de estudios a nivel internacional y nacional, la discusión epistemológica y metodológica es aún un ámbito emergente en nuestra región.

La problematización sobre cómo se construye conocimiento y los posicionamientos epistemológicos de los que derivan métodos, técnicas y procedimientos ha quedado subordinada a la obtención de resultados de la investigación, primando siempre la ponderación de los hallazgos. Ello puede observarse repasando las publicaciones científicas y actas de congresos realizados en la región en Ciencias Sociales, en los últimos años.

En este sentido, por lo tanto, es meritoria la convocatoria de De Prácticas y Discursos, que con este dossier inaugura la posibilidad de debatir especificamente sobre enfoques metodológicos, técnicas, operaciones y, con ello, poner en cuestión a la práctica investigativa y el quehacer científico en Ciencias Sociales.

Para ello, se planteó indagar en los aspectos teóricos y metodológicos involucrados en la investigación, exponer y someter a crítica las estrategias de trabajo investigativo en las distintas disciplinas sociales, los abordajes y técnicas, sus fundamentos epistemológicos y las condiciones de realización de la investigación social en nuestra región.

La atención a los problemas sociales y políticos nos enfrenta, en esta dirección, a la reflexión sobre cómo se definen esos problemas y cómo se produce conocimiento de base para su resolución. Precisamente, la relevancia de la reflexión metodológica radica en cómo se construye/deconstruye conocimiento, sosteniendo esta labor en procedimientos rigurosos, confiables y creativos. Para empezar, el artículo Pensamiento sistémico, complejidad y Ciencias Sociales: las bases epistemológicas de las metodologías participativas, de Alejandro Noboa Silva, alude a las consecuencias de asumir la complejidad como forma de abordaje de los sistemas sociales que implica reconocer que, "como observadores, estamos en el mundo, y el mundo cambia y nos modifica mientras intentamos conocerlo", como fundamento de las metodologías de investigación acción participativas. Este trabajo contiene una propuesta de vinculación propia de las nociones analizadas y una revisión teórica de base para la comprensión de la comple- 
De Prácticas y discursos

Universidad Nacional del Nordeste

Centro de Estudios Sociales

Año 7, Número 9, 2018, Marzo

ISSN 2250-6942

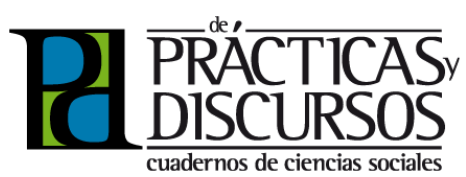

jidad como enfoque, y la investigación acción como estrategia metodológica válida para emprender el conocimiento al servicio de la transformación social.

En Los relatos de vida como recurso de investigación de problemáticas vocacionales, de Elena del C. Moiraghi, se aborda la relevancia de los relatos de vida como estrategias de investigación en Ciencias Sociales en general y de problemáticas vocacionales en particular. Además, presenta una forma de abordaje riguroso y sistemático de relatos de vida referidos a problemáticas vocacionales. Apoyada en una completa y bien estructurada revisión teórica sobre abordajes de historias y relatos de vida, la autora hace evidente la doble reflexividad implicada en el análisis de los casos. Muestra, asimismo, la forma operativa de implementación de la sistematización y el análisis.

El artículo Los modos de la mirada. Aportes para una epistemología crítica de la observación en la investigación social, de Verónica Hendel, presenta un recorrido comparativo de los modos de concebir la observación en el devenir de los estudios sociales. El pasaje de la mirada objetivante -propia de los abordajes tradicionales- a la observación densa y reflexiva y situada -propia de los abordajes actuales críticos- está argumentada sobre la base de una discusión epistemológica que clarifica sobre la reflexividad necesaria para el conocimiento del mundo social. La etnografía se propone, así, como una observación compleja y necesariamente dispuesta a descubrir más.

Por otro lado, en Los problemas de la investigación comparativa sobre la seguridad social en la Argentina (2003-2015), de Susana Hintze y Claudia Danani, se exponen los desafíos de una investigación comparativa, sobre la base de un análisis de los sistemas de protección social de adultos mayores y niños, niñas y adolescentes, tomando el nivel nacional y provincial, y para ello, los de tres provincias argentinas. Las autoras presentan la potencia de este tipo de estudio, aunque también los obstáculos que debieron superar, ante problemas de producción de información institucional y estadistica que detectaron y que los obligaron a rediseñar el estudio inicialmente propuesto. El artículo, además de problematizar sobre el objeto abordado: la protección social en la Argentina, ofrece una reflexión sobre las decisiones metodológicas para el diseño de estrategias alternativas que permitieron responder las preguntas de investigación.

La investigación, como práctica social comprometida con la 
transformación social, subyace en la motivación para investigar y dar a conocer los resultados, propiciar debates que aporten otra mirada de fenómenos recurrentes y ciertamente naturalizados, para fortalecer estrategias colectivas e incidir en la elaboración de políticas públicas; por lo que, en Los usos de la investigaciónacción-participativa (IAP) para el estudio de los riesgos psicosociales en el trabajo. Reflexiones a partir de una experiencia con representantes sindicales y trabajadores, de María Laura Henry, se da cuenta de la experiencia de investigación con trabajadores de Anses, realizada a fin de visibilizar sus padecimientos. Aborda el enfoque metodológico de la investigación acción y la perspectiva teórica de los riesgos psicosociales del trabajo. El estudio disparado por una demanda de una organización sindical conjuga la investigación científica, la praxis social y el involucramiento de los destinatarios de los resultados, descubre las complejidades y la potencia de la coproducción investigativa.

El artículo Observatorio de conflictos sociales del Nordeste Argentino. Abordaje conceptual y metodológico, de Marcelo Graciosi, Maximiliano Román y Ana Rosa Pratesi, por su parte, aborda los fundamentos teóricos y resultados de la implementación de un observatorio de conflictos sociales en la región NEA. Enlazan el enfoque teórico que enfatiza la centralidad del conflicto en la dinámica social con la observación sistemática de hechos de resistencia, haciendo operativa una matriz que permite registrarlos y describirlos. Se presenta un recorrido crítico de estudios sobre la conflictividad social y el fundamento del propio posicionamiento del equipo de investigación. Este trabajo permite ver fundamentos, dispositivos y resultados. De manera contundente, presenta a la práctica investigativa como insumo de la visibilidad de procesos sociales que están ocultos como tales.

Configuraciones militantes contemporáneas. Una propuesta metodológica para pensar el compromiso político, de Fernando Aiziczon, despliega la relación entre producción de conocimiento y compromiso con la acción/intervención en la realidad analizando la práctica militante, integrando tres aspectos a saber: el sujeto militante que encarna o simboliza la acción; la forma organizativa hegemónica para organizar, incentivar y sostener el compromiso y la acción; y el modo de estudio de esa militancia. La discusión sobre fuentes y enfoques se entreteje presentando la imbricación del activismo con el abordaje académico y la complejidad de estudiar un objeto con el que se está involucrado. El 
De Prácticas y discursos

Universidad Nacional del Nordeste

Centro de Estudios Sociales

Año 7, Número 9, 2018, Marzo

ISSN 2250-6942

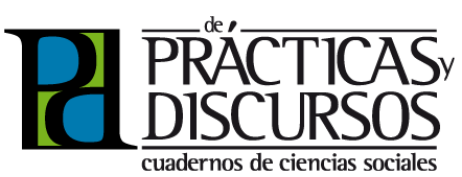

autor establece una tipología de configuraciones militantes en la historia argentina, conjugando las variables expuestas y propone pensar nuevas configuraciones posibles.

En Etnografía colectiva de eventos: la cronotopía paradojal de la Marcha de la Gorra (Córdoba, Argentina), de Andrea BonviIlani, situada como investigadora y militante, la autora presenta el abordaje etnográfico de una manifestación de reclamo (las Marchas de la Gorra en Córdoba) expresando que toda elección metodológica es, a la vez, ético-político. La etnografía se despliega en toda su potencialidad como búsqueda de comprensión de la perspectiva de los actores, por parte de una investigadora también situada, desarrollando una "etnografía colectiva de eventos". La autora describe con precisión todos los registros y participaciones que se integran en la etnografia, posibles en virtud de su principal rasgo: la construcción de vínculos, más que de la aplicación de procedimientos. Se revaloriza en este artículo la etnografía como proceso y como compenetración con el ámbito y los grupos en estudio.

La enseñanza de la investigación, los desafíos de los procesos de enseñanza aprendizaje de la práctica investigativa, es otro de los tópicos abordados por los trabajos que aqui se presentan; por lo que se observará en el artículo Construir el objeto: enseñar metodología desde la praxis investigativa. El caso de Metodología de la Investigación en Ciencias Sociales en las carreras de Sociología y Ciencia Política de la Universidad Nacional de Tierra del Fuego que sus autores, Ariel Farías, Mariano Hermida, Romina Gil, Nadia Pessina y Melisa Urdapilleta, repasan una experiencia de enseñanza de la metodología desde una práctica investigativa activa y analizan dinámicas pedagógicas y didácticas, que parten de la premisa que "la investigación se aprende haciendo". Mediante un exhaustivo recorrido por los momentos, y las actividades realizadas en el proceso, concluyen en la potencia de la implementación del taller de investigación como espacio de reflexión epistémico metodológica.

Así también, El lugar de la teoría en la construcción del objeto de investigación. Decisiones teóricas que involucran un cambio epistemológico, de María Florencia Di Matteo Demirdjian, aborda el lugar de la teoría en la construcción y redefinición del objeto de estudio a propósito de indagar sobre la evaluación de los aprendizajes en instancias de formación preprofesional, sus vinculaciones con la profesión y el proceso formativo del sujeto.

| PAGINA 6 | DOSSIER | 
El artículo aporta significativas reflexiones sobre el oficio de investigador, reconociendo la implicación del sujeto investigador y los vínculos entre la investigación como práctica social situada y los procesos reflexivo/cognitivos del investigador.

En el Cine, identidades y comunidades. Reflexiones metodológicas a partir de una investigación sobre cine e imaginarios sociales en el Mercosur, de Marina Moguillansky, se da cuenta de los imaginarios culturales proyectados en el cine a partir del análisis interpretativo de películas. Desde el enfoque de la sociología de la cultura se presenta el marco teórico que sostiene la potencia del cine como expresión y activo constructor de los imaginarios, en este caso, la elaboración simbólica del proceso de integración regional (Mercosur). La autora expone exhaustivamente la construcción de los instrumentos para interpretación del discurso fílmico, explayándose en los criterios y definiciones operacionales, y discute dificultades de la unidad de observación: el cine latinoamericano. En otro sentido, Investigar la complejidad, asumir la incertidumbre, de Claudia Liliana Perlo, expone el diseño e implementación de una investigación-acción que se apoya en una opción epistémica: hacer operativos los principios de la complejidad y reconocer la incertidumbre presente en todo proceso de conocimiento. El trabajo da cuenta de cómo se abordó un estudio que combina una multiplicidad de métodos y técnicas distinguiendo rigurosidad de rigidez (como postula Morin) y, con ello, extender las posibilidades metodológicas. La autora expone una postura ontoepistemológica y ética desde el imperativo de integrar (enfoques, técnicas y métodos; ciencia, juego y arte; intelecto y sensibilidad) para ampliar los límites de la forma hegemónica de producción de conocimiento.

Los artículos de este dossier, lejos de presentar un panorama homogéneo, nos acercan a las enormes posibilidades de la producción de conocimiento científico en Ciencias Sociales, campo siempre atravesado por la tensión entre la explicación y la comprensión como formas de abordaje metodológico.

La aceptación acrítica de este dualismo, o el encuadramiento a una forma teórico- metodológica (el objetivismo o el subjetivismo por caso, o el abordaje según un único método de aprehensión explicativo o comprensivo), puede pensarse como un paradigma en decadencia a partir de los años ochenta en que se incorpora el reconocimiento de las reflexividades en juego en el proceso de conocimiento. Se evidencia así un tránsito de la 
ciencia social desde de la dualidad hacia una integración/complementación de abordajes, en un movimiento que coloca en el centro del trabajo investigativo antropo-sociológico la misión de "envolver al fenómeno objeto de estudio" como forma de saldar la pseudo distinción entre sujeto cognoscente y sujetos objetos de conocimiento (Guber, 2004; Morin, 2000).

La integración complementaria de las formas de aprensión implica la instauración de un nuevo paradigma en el cual el momento objetivo y el subjetivo son dos instancias de un mismo abordaje, que encuentran la posibilidad de objetivar en el reconocimiento de la subjetividad.

Como lo expresa Bourdieu (1999), "contra la antigua distinción de Dilthey hay que plantear que comprender y explicar son una misma cosa".

La investigación en sus dimensiones epistemológica, técnicometodológica y política se presenta en este dossier como un objeto de estudio fértil. Sin dudas, continuar con esta iniciativa contribuirá a fortalecer el campo de abordaje de la metodología, y de las Ciencias Sociales en la región y el país.

\section{BIBLIOGRAFÍA}

BOURDIEU, P. (DIR.) (1999). Comprender en La miseria del Mundo ( $1^{\text {a }}$ ed. en francés de 1993). Madrid: Fondo de Cultura Económica.

GUBER, R. (2004). El salvaje metropolitano. Reconstrucción del conocimiento social en el trabajo de campo (1a ed. de 1991). Buenos Aires: Paidós.

MORIN, E. (2000). Sociología. Madrid: Tecnos. 